\title{
Study of applicability of the methods of medium and long term load forecasting in new economy normal state
}

\author{
Li-Ning Zhao ${ }^{1, \text { a }}$, Zong-Chuan Zhou ${ }^{1, b}$, \\ Ya-Peng Zhang ${ }^{1, \mathrm{c}}$ and Meng-Kai Wu $\mathrm{W}^{2, \mathrm{~d}, \dagger}$ \\ ${ }^{1}$ State Grid Ningxia Electric Power Eco-Tech Research Institute \\ Yinchuan, China \\ ${ }^{2}$ North China Electric Power University \\ Beijing, China \\ ${ }^{a}$ E-mail:In_Zhao@yeah.net, ${ }^{b} E$-mail: generally@163.com, \\ ${ }^{c}$ E-mail:524267710@qq.com, ${ }^{\dagger},{ }^{d}$ E-mail:963261896@qq.com.
}

\begin{abstract}
Medium and long term load forecasting is important for power system planning and construction. Affected by the New Economy normal and other relevant factors, various regions of China have entered a new period of economic development mode and industrial structure changing. Load structure and load growth pattern, to a certain extent, has changed, which undoubtedly makes the long-term load forecasting more complex and uncertain. To study the models and methods of Medium and long term load forecasting in the market environment is more important. This paper deeply analyzes the new economy normal state's features, refines the factors which can properly characterize the new normal state's changing, and applies them to the medium and long term load forecasting's models; in accordance with the actual data of one province, uses a variety of methods and models to calculate and compare the predictions, and gets the applicability of long-term load forecasting model in new economy normal state.
\end{abstract}

Keywords: Medium And Long Term Load Forecasting; New Economy Normal State; Electricity Elasticity Coefficient; Regression Analysis.

\section{Introduction}

In recent years, as China's economy has entered a new normal state with a lowspeed development, by the effect of global overcapacity, low-carbon economy and energy saving policies, load structure and growth pattern has changed greatly, with the implementation of One Belt And One Road, the excess capacity will be effectively averted. The load level will be enhanced in relevant areas. In the context of the accelerating electricity system reform, by the effect of transmission and distribution tariff reform and vending side opens, enterprise's energy cost will be further reduced, which can also enhance the load level. New normal state's complexities have put forward new requirements for medium and long term load forecasting models and methods. 
Many domestic and foreign experts and scholars also conducted research on medium and long term load forecasting[1], but mostly based on the traditional environment[2]. Under the new normal state, the applicability of medium and long term load forecasting models will be changed to some degree.

Firstly, the new normal state is analyzed deeply, and the factors accurately reflecting the features of new normal state are refined, which are applied to the models and methods of the medium and long term load forecasting. Secondly, according to the actual data of Yunnan province, the paper uses various methods to predict medium and long term load as well as analyzes the error. Finally, based on the results of error analysis, the applicability of all kinds of predictive models in new normal state is got.

\section{Situations of Market Environment}

Since 2014, China's economy entered a low-speed development of the "new normal", by the impact of overcapacity, in many parts of the country, the big industry was severely affected, which has a larger proportion of electricity structure. Enterprises surviving environment deteriorated. Production and electricity consumption declined and spread to other industries[3].

With the "CPC Central Committee and State Council on further deepening of the power system" introducing, the process of China's electricity marketing begins to accelerate. Transmission and distribution tariff reform and vending side opening starts in succession.

In order to promote industrial restructuring and upgrading, the government introduced the energy conservation policy, and came up with the "One Belt And One Road", to resolve excess capacity and promote economic growth. This will largely affect the structure of the load and the load growth patterns around the country[4].

\section{Major Influencing Factors}

\subsection{Macroeconomic factors}

The level of economic development has fundamental and overall impact on the load[5].We use GDP as an indicator to measure the economic environmental changing. The GDP growth slows, the economy slows down, the industry production activities slow, while the electricity consumption growth slows.

\subsection{Power system reform}

With the "CPC Central Committee and State Council on further deepening of the power system" introducing, the process of China's transmission and distribution 
tariff reform and vending side opening begins to accelerate. With the vending side further opening, the electricity prices will be reduced because of oversupply in Electricity Market. Enterprises' energy costs will be decline. Enterprises will expand production, and the electricity consumption will increase[6]. Some enterprises have relatively fixed electricity using period due to the operating characteristics. Their consumption enhancing, to some extent will make the peak-valley difference expand. We use the electricity prices as the index to measure the impact of power system reform.

\subsection{Policy factors}

In 2013, the government came up with the "One Belt And One Road". It will rely on the existing resource allocation platform to effectively resolve the domestic overcapacity, surplus of foreign exchange assets and other issues. It will further promote economic development. To a certain extent, it will slow the decline of the load growth rate.

Policies consisting mainly of large-scale infrastructure investment will lead to short-term load levels lifting in some areas[7]. We characterize this using the total fixed asset investment.

\section{Medium and Long Term Load Forecasting Models and Methods}

This paper selects representatives of the traditional methods to calculate and compare the prediction error, to analyze the applicability of different prediction methods under the new normality.

\subsection{Trend extrapolation}

Trend extrapolation is a method fits load variation to extrapolate. Generally it applies to the occasions in which the trend is obvious, and the random factors have little effect[8]. Typical methods are Growth curve Extrapolating (GCE) and Exponential Smoothing (ESE).

Growth curve Extrapolating (GCE) is a method using some kind of growth curve, to fit historical load data, and establish the model to describe the load development process. Exponential smoothing Extrapolating (ESE) is a timing analysis. Its predictive value is the weighted arithmetic average of the historical data. And the recent weight is bigger than the old one.

\subsection{Regression analysis}

Regression analysis (RA) is to make regression analysis between load and other related variables (GDP, etc.). And based on the predicted value of other relevant variables, make load forecasting. It generally applies to the case in which the 
relationship between the electricity consumption and relevant factors (GDP, etc.) is more stable.

\subsection{Electricity elasticity coefficient method}

Electricity elasticity coefficient method (EEC) first calculates the electricity elasticity coefficient based on historical electricity using data and historical GDP growth rate. Then use some method to predict the future electricity elasticity coefficient and the GDP growth rate. Finally, calculate future consumption growth rate and the future electricity consumption. Different from the traditional environment, in market environment, affected by the regional macroeconomic factors, industry adjustment policy factors, electricity elasticity coefficient is often volatile. Because electricity elasticity coefficient method has requirement for higher accuracy of the GDP growth forecast, it hasn't great applicability.

\subsection{Method of unit consumption of industrial output value}

Method of unit consumption of industrial output value (UCIO) is to calculate unit consumption based on the historical electricity and production data, and calculate the future electricity consumption based on the future output value.

This method is generally applicable to the case in which industrial output predictions have little change or a higher precision.

Different from traditional environment, in the new normality, unit consumption of industrial output value in some areas may have greater changes. Therefore, under the new situation, the applicability of the method will decrease.

\section{Cases Operators of Typical Areas}

Take Yunnan province for example, according to the province's total GDP from 2005 to 2011, the province's electricity consumption from 2007 to 2010 and various industrial electricity consumption raw data, use trend extrapolation, regression model, the industrial output value of unit consumption, predict the electricity consumption from 2012 to 2014, the results and prediction errors are following: 
Table 1. Various methods predicting results and error comparison in Yunnan

\begin{tabular}{cccc}
\hline Year & 2012 & 2013 & 2014 \\
\hline actual value (hundred million kWh) & 1315.9 & 1459.81 & 1529.38 \\
\hline GCE(hundred million $\mathrm{kWh})$ & 1320.23 & 1489.25 & 1679.91 \\
Deviations & $0.33 \%$ & $2.02 \%$ & $9.84 \%$ \\
\hline ESE(hundred million kWh) & 1238 & 1339 & 1439 \\
Deviations & $-5.92 \%$ & $-8.28 \%$ & $-5.91 \%$ \\
\hline RA(hundred million $\mathrm{kWh)}$ & 1343.03 & 1535.65 & 1760.04 \\
Deviations & $2.06 \%$ & $5.20 \%$ & $15.08 \%$ \\
\hline UCIO(hundred million $\mathrm{kWh})$ & 1200.15 & 1405.58 & 1646.21 \\
Deviations & $-8.80 \%$ & $-3.71 \%$ & $7.64 \%$ \\
\hline EEC(hundred million $\mathrm{kWh})$ & 1361.31 & 1526.2 & 1630.1 \\
Deviations & $3.45 \%$ & $4.54 \%$ & $6.58 \%$ \\
\hline
\end{tabular}

We can find this by analyzing all kinds of methods' predicting results.

(1)In the predictions of various methods in Yunnan, the predicting deviations of the Growth curve Extrapolating for 2012 is small. The reason is that the change of electricity consumption growth rate for 2012 is small relative to the approaching years, 2013, too. But the electricity consumption growth rate in 2014 has a large decline, and leads to a large error. Generally, it applies to the case with load steady growth. In new normality, the electricity consumption growth rate may have a greater volatility.

(2)The predicting deviations of the Regression analysis for 2014 is large, because of forecast Yunnan's GDP growth rate using the Growth curve Extrapolating, which assumes that the growth rate of GDP changes little. The reality is that the GDP growth rate of Yunnan in 2014 has a great reduction, compared with 2013 and 2014. This leads to a sudden increase of the prediction errors in 2014. The RA has a high dependency for selected factors (GDP etc.).

(3) The predictions of UCIO for 2014 is larger, as a result of prices for aluminum inverted at home and abroad. Aluminum import surges (increased 200\%). Domestic aluminum smelting industry downturn(The loss increasing 3.24 billion yuan). It led deterioration of business environment for electrolytic aluminum enterprises in Yunnan where the electrolytic aluminum industry has a large proportion of electricity consumption. And their electricity consumption reduced. The unit electricity consumption of secondary industry dropped. As a result, the deviations of the UCIO were large.The UCIO has a high dependency for the unit electricity consumption.

(4)The predictions of the ESE is smaller from 2012 to 2014 than the actual value. And the deviations are stable. Because of the weighted arithmetic average of the historical data and the recent weight bigger than the old one, it can effectively reflect the impact of the recent economic situation changing. 


\section{Conclusions}

This paper deeply analyzes the new economy normal state's features, refines the factors which can properly characterize the new normal state's changing, and applies them to the medium and long term load forecasting models; in accordance with the actual data of Yunnan uses a variety of methods and models to calculate and compare the predictions, and gets the applicability of long-term load forecasting model in new economy normal state.

\section{References}

1. Z. G. Qi, X H Yuan and J W Wang A new method for mid-long term load forecasting of power systems J. Power System Engineering Vol 18 p 39-41 (2002). (In Chinese)

2. P. Jiang and P. Ju. J. Automation of Electric Power Systems Vol 19 p 11-17 (1995). (In Chinese)

3. D. Q. Zhou Application of improved gray verhulst model in middle and long term load forecasting J. Power System Technology Vol 33 No 18 p 124-127 (2009). (In Chinese)

4. Franklin L. Quilumba, Wei-Jen Lee. Using smart meter data to improve the accuracy of intraday load forecasting considering customer behavior similarities J. Smart Grid Vol 6 No 2 p 911-918 (2015).

5. C. Q. Kang, Q. Xia and B M Zhang J. Automation of Electric Power Systems Vol 28 No 17 p 1-11 (2004). (In Chinese)

6. T. Senjyu, H. Takara; K. Uezato, T. Funabashi One-hour-ahead load forecasting using neural network J. IEEE Transactions on Power Systems Vol 17 No 1 p 113 - 118 (2002).

7. Tao Hong, Jason Wilson, Jingrui XieLong term probabilistic load forecasting and normalization with hourly information J. IEEE Transactions on Smart Grid Vol 5 No 1 p 456 - 462 (2014). (In Chinese)

8. X. K. Jin, Z. J. He and Z. Q. Liu Mid-long term load forecasting based on cloud theory J. Modern Electric Power Vol 28 p 40-43 (2011). (In Chinese) 